\title{
STOCHASTIC EULERIAN LAGRANGIAN METHODS FOR FLUID-STRUCTURE INTERACTIONS WITH THERMAL FLUCTUATIONS
}

\author{
PAUL J. ATZBERGER *
}

\begin{abstract}
We present approaches for the study of fluid-structure interactions subject to thermal fluctuations. A mixed mechanical description is utilized combining Eulerian and Lagrangian reference frames. We establish general conditions for operators coupling these descriptions. Stochastic driving fields for the formalism are derived using principles from statistical mechanics. The stochastic differential equations of the formalism are found to exhibit significant stiffness in some physical regimes. To cope with this issue, we derive reduced stochastic differential equations for several physical regimes. We also present stochastic numerical methods for each regime to approximate the fluid-structure dynamics and to generate efficiently the required stochastic driving fields. To validate the methodology in each regime, we perform analysis of the invariant probability distribution of the stochastic dynamics of the fluid-structure formalism. We compare this analysis with results from statistical mechanics. To further demonstrate the applicability of the methodology, we perform computational studies for spherical particles having translational and rotational degrees of freedom. We compare these studies with results from fluid mechanics. The presented approach provides for fluidstructure systems a set of rather general computational methods for treating consistently structure mechanics, hydrodynamic coupling, and thermal fluctuations.
\end{abstract}

Key words. Fluid-Structure Interaction, Statistical Mechanics, Fluid Dynamics, Thermal Fluctuations, Fluctuating Hydrodynamics, Stochastic Eulerian Lagrangian Method, SELM.

1. Introduction. The development of analytic and computational approaches for the study of fluid-structure interactions has a rich history. Motivations for past work in this area include the study of aerodynamic oscillations induced in airplane wings and propellers [22, 24], the study of animal locomotion including swimming and insect flight [44, 38, 51], and the study of physiological problems such as blood flow through heart valves [30, 49, 27]. A central challenge in work on these applications has been to develop descriptions which capture essential features of the fluid structure interactions while introducing approximations which facilitate analysis and the development of tractable numerical methods [22, 45]. Many such challenges remain and this area of research is still very active [30, 13, 45, 12]. Recent scientific and technological advances motivate the study of fluid-structure interactions in new physical regimes often involving very small length scales [57, 64, 15, 46]. At sufficiently small length scales thermal fluctuations play an important role and pose additional challenges in the study of fluid-structure systems.

Significant past work has been done on the formulation of descriptions of fluidstructure interactions subject to thermal fluctuations. Many of these analytic and numerical approaches originate from the polymer physics community $[19,23,52,11]$. To obtain descriptions tractable for analysis and numerical simulation, these approaches typically place an emphasis on approximations which retain only the structure degrees of freedom. This often results in significant simplifications in the descriptions and in significant computational savings. This eliminates the many degrees of freedom associated with the fluid and avoids having to resolve the potentially intricate and stiff stochastic dynamics of the fluid. These approaches have worked especially well for the study of bulk phenomena in free solution and the study of complex fluids and soft

*University of California, Department of Mathematics , Santa Barbara, CA 93106; e-mail: atzberg@math.ucsb.edu; phone: 805-893-3239; Work supported by NSF CAREER Grant DMS 0956210. http://www.math.ucsb.edu/ atzberg/ 
materials $[52,19,37]$.

Recent applications arising in the sciences and in technological fields present situations in which resolving the dynamics of the fluid may be important and even advantageous both for modeling and computation. This includes modeling the spectroscopic responses of biological materials [65, 28,43], studying transport in microfluidic and nanofluidic devices [57, 47], and investigating dynamics in biological systems [2, 17]. There are also other motivations for representing the fluid explicitly and resolving its stochastic dynamics. This includes the development of hybrid fluid-particle models in which thermal fluctuations mediate important effects when coupling continuum and particle descriptions $[18,20]$, the study of hydrodynamic coupling and diffusion in the vicinity of surfaces having complicated geometries [57], and the study of systems in which there are many interacting mechanical structures $[7,50,49]$. To facilitate the development of methods for studying such phenomena in fluid-structure systems, we present a rather general formalism which captures essential features of the coupled stochastic dynamics of the fluid and structures.

To model the fluid-structure system, a mechanical description is utilized involving both Eulerian and Lagrangian reference frames. Such mixed descriptions arise rather naturally, since it is often convenient to describe the structure configurations in a Lagrangian reference frame while it is convenient to describe the fluid in an Eulerian reference frame. In practice, this presents a number of challenges for analysis and numerical studies. A central issue concerns how to couple the descriptions to represent accurately the fluid-structure interactions, while obtaining a coupled description which can be treated efficiently by numerical methods. Another important issue concerns how to account properly for thermal fluctuations in such approximate descriptions. This must be done carefully to be consistent with statistical mechanics. A third issue concerns the development of efficient computational methods. This requires discretizations of the stochastic differential equations and the development of efficient methods for numerical integration and stochastic field generation.

We present a set of approaches to address these issues. The formalism and general conditions for the operators which couple the Eulerian and Lagrangian descriptions are presented in Section 2. We discuss simplified descriptions of the fluid-structure system in different physical regimes in Section 3. A derivation of the stochastic driving fields used to represent the thermal fluctuations is also presented in Section 3. Stochastic numerical methods are discussed for the approximation of the stochastic dynamics and generation of stochastic fields in Sections 4. To validate the methodology, we perform in each regime analysis of the invariant probability distribution of the stochastic dynamics of the fluid-structure formalism. We compare this analysis with results from statistical mechanics in Section 5. To demonstrate the applicability of the methodology, we perform computational studies for spherical particles having translational and rotational degrees of freedom. We compare these computational studies with results from fluid mechanics in Section 6 .

It should be mentioned that related computational methods have been introduced for the study of fluid-structure interactions [50, 3, 10, 12, 63, 45, 67, 33, 41]. In recent papers, significant work also has been done toward incorporating the role of thermal fluctuations $[6,14,9,21,7]$. This includes the Stochastic Immersed Boundary Method [6], Fluctuating Immersed Material Dynamics [14], Computational Fluctuating Fluid Dynamics [9, 21], and Accelerated Stokesian Dynamics [7]. The formalism presented here can be regarded in part as a generalization of these approaches. It is expected that many of the presented results can be applied to further justify and val- 


\section{Lagrangian Reference Frame}

\section{Eulerian Reference Frame}

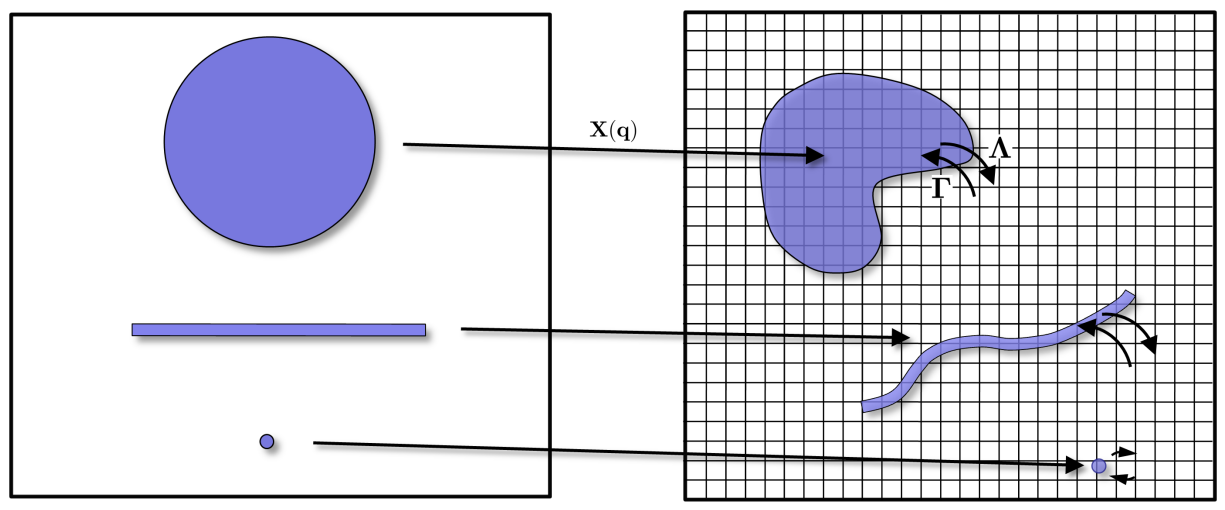

FIG. 2.1. The description of the fluid-structure system utilizes both Eulerian and Lagrangian reference frames. The structure mechanics are often most naturally described using a Lagrangian reference frame. The fluid mechanics are often most naturally described using an Eulerian reference frame. The mapping $\mathbf{X}(\mathbf{q})$ relates the Lagrangian reference frame to the Eulerian reference frame. The operator $\Gamma$ prescribes how structures are to be coupled to the fluid. The operator $\Lambda$ prescribes how the fluid is to be coupled to the structures. A variety of fluid-structure interactions can be represented in this way. This includes rigid and deformable bodies, membrane structures, polymeric structures, or point particles.

idate these methods and to provide further extensions. The formalism presented here provides a rather general framework for the development of computational methods for applications requiring a consistent treatment of structure mechanics, hydrodynamic coupling, and thermal fluctuations.

2. Summary of the Stochastic Eulerian Lagrangian Method . We summarize here the Stochastic Eulerian Lagrangian Method, abbreviated as SELM. We present the general formalism and a number of alternative descriptions of the fluidstructure system. In many situations the stochastic differential equations for the full fluid-structure dynamics exhibits stiffness. To cope with this issue and to develop efficient numerical methods, simplified descriptions are discussed for various physical regimes. A more detailed discussion and derivation of SELM and the reduced equations in each of the physical regimes is given in Section 3.

To study the dynamics of fluid-structure interactions in the presence of thermal fluctuations, we utilize a mechanical description involving Eulerian and Lagrangian reference frames. Such mixed descriptions arise rather naturally, since it is often convenient to describe the structure configurations in a Lagrangian reference frame while it is convenient to describe the fluid in an Eulerian reference frame. In principle more general descriptions using other reference frames could also be considered. Descriptions for fluid-structure systems having these features can be described rather generally by the following dynamic equations

$$
\begin{aligned}
\rho \frac{d \mathbf{u}}{d t} & =\mathcal{L} \mathbf{u}+\Lambda[\Upsilon(\mathbf{v}-\Gamma \mathbf{u})]+\lambda+\mathbf{f}_{\mathrm{thm}} \\
m \frac{d \mathbf{v}}{d t} & =-\Upsilon(\mathbf{v}-\Gamma \mathbf{u})-\nabla_{\mathbf{X}} \Phi[\mathbf{X}]+\zeta+\mathbf{F}_{\mathrm{thm}} \\
\frac{d \mathbf{X}}{d t} & =\mathbf{v}
\end{aligned}
$$


The $\mathbf{u}$ denotes the velocity of the fluid, $\rho$ the uniform fluid density. The $\mathbf{X}$ denotes the configuration of the structure and $\mathbf{v}$ the velocity of the structure. The mass of the structure is denoted by $m$. To simplify the presentation we treat here only the case when $\rho$ and $m$ are constant, but with some modifications these could also be treated as variable. The $\lambda, \zeta$ are Lagrange multipliers for imposed constraints, such as incompressibility of the fluid or a rigid body constraint of a structure. The operator $\mathcal{L}$ is used to account for dissipation in the fluid, such as associated with Newtonian fluid stresses [1]. To account for how the fluid and structures are coupled, a few general operators are introduced, $\Gamma, \Upsilon, \Lambda$.

The linear operators $\Gamma, \Lambda, \Upsilon$ are used to model the fluid-structure coupling. The $\Gamma$ operator describes how a structure depends on the fluid flow while $-\Upsilon$ is a negative definite dissipative operator describing the viscous interactions coupling the structure to the fluid. We assume throughout that this dissipative operator is symmetric, $\Upsilon=\Upsilon^{T}$. The linear operator $\Lambda$ is used to attribute a spatial location for the viscous interactions between the structure and fluid. The linear operators are assumed to have dependence only on the configuration degrees of freedom $\Gamma=\Gamma[\mathbf{X}], \Lambda=\Lambda[\mathbf{X}]$. We assume further that $\Upsilon$ does not have any dependence on $\mathbf{X}$.

To account for the mechanics of structures, $\Phi[\mathbf{X}]$ denotes the potential energy of the configuration $\mathbf{X}$. The total energy associated with this fluid-structure system is given by

$$
E[\mathbf{u}, \mathbf{v}, \mathbf{X}]=\int_{\Omega} \frac{1}{2} \rho|\mathbf{u}(\mathbf{y})|^{2} d \mathbf{y}+\frac{1}{2} m \mathbf{v}^{2}+\Phi[\mathbf{X}] .
$$

The first two terms give the kinetic energy of the fluid and structures. The last term gives the potential energy of the structures.

As we shall discuss, it is natural to consider coupling operators $\Lambda$ and $\Gamma$ which are adjoint in the sense

$$
\int_{\mathcal{S}}(\Gamma \mathbf{u})(\mathbf{q}) \cdot \mathbf{v}(\mathbf{q}) d \mathbf{q}=\int_{\Omega} \mathbf{u}(\mathbf{x}) \cdot(\Lambda \mathbf{v})(\mathbf{x}) d \mathbf{x}
$$

for any $\mathbf{u}$ and $\mathbf{v}$. The $\mathcal{S}$ and $\Omega$ denote the spaces used to parameterize respectively the structures and the fluid. We denote such an adjoint by $\Lambda=\Gamma^{\dagger}$ or $\Gamma=\Lambda^{\dagger}$. This adjoint condition can be shown to have the important consequence that the fluid-structure coupling conserves energy when $\Upsilon \rightarrow \infty$ in the inviscid and zero temperature limit.

In practice, the conditions discussed above can be relaxed somewhat. For our present purposes these conditions help simplify the presentation. Each of these operators will be discussed in more detail below.

To account for thermal fluctuations, a random force density $\mathbf{f}_{\text {thm }}$ is introduced in the fluid equations and $\mathbf{F}_{\text {thm }}$ in the structure equations. These account for spontaneous changes in the system momentum which occurs as a result of the influence of unresolved microscopic degrees of freedom and unresolved events occurring in the fluid and in the fluid-structure interactions.

The thermal fluctuations consistent with the form of the total energy and relaxation dynamics of the system are taken into account by the introduction of stochastic driving fields in the momentum equations of the fluid and structures. The stochastic driving fields are taken to be Gaussian processes with mean zero and with $\delta$-correlation in time [54]. By the fluctuation-dissipation principle [54] these have covariances given by

$$
\left\langle\mathbf{f}_{\mathrm{thm}}(s) \mathbf{f}_{\mathrm{thm}}^{T}(t)\right\rangle=-\left(2 k_{B} T\right)(\mathcal{L}-\Lambda \Upsilon \Gamma) \delta(t-s)
$$




$$
\begin{aligned}
\left\langle\mathbf{F}_{\text {thm }}(s) \mathbf{F}_{\text {thm }}^{T}(t)\right\rangle & =\left(2 k_{B} T\right) \Upsilon \delta(t-s) \\
\left\langle\mathbf{f}_{\text {thm }}(s) \mathbf{F}_{\text {thm }}^{T}(t)\right\rangle & =-\left(2 k_{B} T\right) \Lambda \Upsilon \delta(t-s) .
\end{aligned}
$$

We have used that $\Gamma=\Lambda^{\dagger}$ and $\Upsilon=\Upsilon^{T}$. We remark that the notation $\mathbf{g h}^{T}$ which is used for the covariance operators should be interpreted as the tensor product. This notation is meant to suggest the analogue to the outer-product operation which holds in the discrete setting [5]. A more detailed discussion and derivation of the thermal fluctuations is given in Section 3.

It is important to mention that some care must be taken when using the above formalism in practice and when choosing operators. An important issue concerns the treatment of the material derivative of the fluid, $d \mathbf{u} / d t=\partial \mathbf{u} / \partial t+\mathbf{u} \cdot \nabla \mathbf{u}$. For stochastic systems the field $\mathbf{u}$ is often highly irregular and not defined in a point-wise sense, but rather only in the sense of a generalized function (distribution) $[16,40]$. This presents issues in how to define the non-linear term arising in the material derivative, which appears to require point-wise values of $\mathbf{u}$. For such irregular velocity fields, this also calls into question the applicability of the theorems typically used to derive the differential equations from the conservation laws. For instance, for such velocity fields the fluid material body may no longer exhibit smooth deformations over time.

There are a number of ways to deal with this issue. The first is to consider a regularization of the fluid stresses, which are typically the source of irregularity, see equation 2.6. This can be motivated by the fact that the fluid stress tensors typically considered in continuum mechanics are expected to become inaccurate at molecular length-scales. Ideally, from molecular models of the fluid the small-length scale (large wave-number) responses of the fluid could be determined and provide a justified regularization. For instance, this could provide an alternative to using responses based on Newtonian stresses for all length-scales. For the SELM formalism, this would simply correspond to using for $\mathcal{L}$ an alternative to the dissipative operator based on Newtonian stresses. The second more easily implemented approach is simply to work with the linearized material derivative, which still retains many of the essential features of the fluid dynamics and is useful for many applications [6].

In this initial presentation of SELM, we shall take the latter approach and treat $d \mathbf{u} / d t=\partial \mathbf{u} / \partial t$. This provides a rather general description of fluid-structure systems which incorporate the role of thermal fluctuations. From this initial formalism of SELM, we shall derive a number of simplified descriptions for various physical regimes. These simplified descriptions for each regime tend to yield less stiff differential equations and have other features making them useful in the development of efficient stochastic numerical methods for the formalism.

2.1. Regime I. We now consider the regime in which the full dynamics of the fluid-structure system are retained but reformulated in terms of a field describing the total momentum of the fluid-structure system at a given spatial location. This description is more convenient to work with in practice since it results in simplifications in the stochastic driving fields. For this purpose we define

$$
\mathbf{p}(\mathbf{x}, t)=\rho \mathbf{u}(\mathbf{x}, t)+\Lambda[m \mathbf{v}(t)](\mathbf{x}) .
$$

The operator $\Lambda$ is used to give the distribution in space of the momentum associated with the structures for given configuration $\mathbf{X}(t)$. Using this approach, the fluidstructure dynamics are described by

$$
\frac{d \mathbf{p}}{d t}=\mathcal{L} \mathbf{u}+\Lambda\left[-\nabla_{\mathbf{X}} \Phi(\mathbf{X})\right]+\left(\nabla_{\mathbf{X}} \Lambda[m \mathbf{v}]\right) \cdot \mathbf{v}+\lambda+\mathbf{g}_{\mathrm{thm}}
$$




$$
\begin{aligned}
m \frac{d \mathbf{v}}{d t} & =-\Upsilon(\mathbf{v}-\Gamma \mathbf{u})-\nabla_{\mathbf{X}} \Phi(\mathbf{X})+\zeta+\mathbf{F}_{\text {thm }} \\
\frac{d \mathbf{X}}{d t} & =\mathbf{v}
\end{aligned}
$$

where $\mathbf{u}=\rho^{-1}(\mathbf{p}-\Lambda[m \mathbf{v}])$ and $\mathbf{g}_{\mathrm{thm}}=\mathbf{f}_{\mathrm{thm}}+\Lambda\left[\mathbf{F}_{\mathrm{thm}}\right]$. The third term in the first equation arises from the dependence of $\Lambda$ on the configuration of the structures, $\Lambda[m \mathbf{v}]=(\Lambda[X])[m \mathbf{v}]$. The Lagrange multipliers for imposed constraints are denoted by $\lambda, \zeta$. For the constraints, we use rather liberally the notation with the Lagrange multipliers denoted here not necessarily assumed to be equal to the previous definition. The stochastic driving fields are again Gaussian with mean zero and $\delta$-correlation in time [54]. The stochastic driving fields have the covariance structure given by

$$
\begin{aligned}
\left\langle\mathbf{g}_{\text {thm }}(s) \mathbf{g}_{\text {thm }}^{T}(t)\right\rangle & =-\left(2 k_{B} T\right) \mathcal{L} \delta(t-s) \\
\left\langle\mathbf{F}_{\text {thm }}(s) \mathbf{F}_{\text {thm }}^{T}(t)\right\rangle & =\left(2 k_{B} T\right) \Upsilon \delta(t-s) \\
\left\langle\mathbf{g}_{\text {thm }}(s) \mathbf{F}_{\text {thm }}^{T}(t)\right\rangle & =0
\end{aligned}
$$

This formulation has the convenient feature that the stochastic driving fields become independent. This is a consequence of using the field for the total momentum for which the dissipative exchange of momentum between the fluid and structure no longer arises. In the equations for the total momentum, the only source of dissipation remaining occurs from the stresses of the fluid. This approach simplifies the effort required to generate numerically the stochastic driving fields and will be used throughout.

2.2. Regime II. We now consider a regime in which the formalism can be simplified significantly. In many situations, inertial effects often play a relatively minor role in the structure dynamics as a consequence of the small mass of the structure relative to the displaced fluid or as a consequence of viscosity of the solvent fluid [31, 42]. In such a regime, the relatively rapid dynamics associated with the momentum of the structures often presents a source of stiffness in numerical calculations. To cope with these issues we consider a reduction of the stochastic dynamics of the system in which the structure momentum is eliminated from the description. In particular, we consider the regime in which $m \ll \rho \ell^{3}$. The $\ell$ denotes a length-scale characteristic of the size of the immersed structure and associated flow field of the fluid. In the limit $m \rightarrow 0$, the fluid-structure dynamics are governed by

$$
\begin{aligned}
\frac{d \mathbf{p}}{d t} & =\rho^{-1} \mathcal{L} \mathbf{p}+\Lambda\left[-\nabla_{\mathbf{X}} \Phi(\mathbf{X})\right]+\left(\nabla_{\mathbf{X}} \cdot \Lambda\right) k_{B} T+\lambda+\mathbf{g}_{\mathrm{thm}} \\
\frac{d \mathbf{X}}{d t} & =\rho^{-1} \Gamma \mathbf{p}+\Upsilon^{-1}\left[-\nabla_{\mathbf{X}} \Phi(\mathbf{X})\right]+\zeta+\mathbf{G}_{\mathrm{thm}} .
\end{aligned}
$$

In the notation $\nabla_{\mathbf{X}} \cdot \Lambda=\operatorname{Tr}\left[\nabla_{\mathbf{X}} \Lambda\right]$. This term arises from the thermal fluctuations associated with the momentum of the structures, which have been eliminated from the description. This term plays an important role in the system when the phasespace dynamics of $(\mathbf{p}, \mathbf{X})$ has an associated vector field which is compressible. When considering the Liouville equation on phase-space, this term accounts for local changes of the phase-space volume which occurs as the configuration of the structure changes under the dynamics of the reduced description [62]. For a more detailed discussion see Section 5.2. The stochastic driving fields $\mathbf{g}_{\mathrm{thm}}, \mathbf{G}_{\mathrm{thm}}$ are again Gaussian with mean zero and with $\delta$-correlation in time [54]. The stochastic driving fields have the 
covariance structure given by

$$
\begin{aligned}
\left\langle\mathbf{g}_{\mathrm{thm}}(s) \mathbf{g}_{\mathrm{thm}}^{T}(t)\right\rangle & =-\left(2 k_{B} T\right) \mathcal{L} \delta(t-s) \\
\left\langle\mathbf{G}_{\mathrm{thm}}(s) \mathbf{G}_{\mathrm{thm}}^{T}(t)\right\rangle & =\left(2 k_{B} T\right) \Upsilon^{-1} \delta(t-s) \\
\left\langle\mathbf{g}_{\mathrm{thm}}(s) \mathbf{G}_{\mathrm{thm}}^{T}(t)\right\rangle & =0 .
\end{aligned}
$$

A more detailed discussion and derivation of the equations in this regime is given in Section 3.2 .

2.3. Regime III. The description of the fluid-structure system can be further simplified by considering the viscous coupling between the fluid and structures in the limit $\Upsilon \rightarrow \infty$. In this case, the fluid-structure dynamics are given by

$$
\begin{aligned}
\frac{d \mathbf{p}}{d t} & =\rho^{-1} \mathcal{L} \mathbf{p}+\Lambda\left[-\nabla_{\mathbf{X}} \Phi(\mathbf{X})\right]+\left(\nabla_{\mathbf{X}} \cdot \Lambda\right) k_{B} T+\lambda+\mathbf{g}_{\mathrm{thm}} \\
\frac{d \mathbf{X}}{d t} & =\rho^{-1} \Gamma \mathbf{p} \\
\left\langle\mathbf{g}_{\text {thm }}(s) \mathbf{g}_{\text {thm }}^{T}(t)\right\rangle & =-\left(2 k_{B} T\right) \mathcal{L} \delta(t-s) .
\end{aligned}
$$

In the notation $\nabla_{\mathbf{X}} \cdot \Lambda=\operatorname{Tr}\left[\nabla_{\mathbf{X}} \Lambda\right]$. A more detailed discussion and derivation of the equations in this regime in given in Section 3.3.

2.4. Regime IV. The description of the fluid-structure system can be further simplified by considering for the fluid the viscous limit in which $\mu \rightarrow \infty$. In this regime only the structure dynamics remain and can be shown to be given by

$$
\begin{aligned}
\frac{d \mathbf{X}}{d t} & =H_{\text {SELM }}\left[-\nabla_{\mathbf{X}} \Phi(\mathbf{X})\right]+\left(\nabla_{\mathbf{X}} \cdot H_{\text {SELM }}\right) k_{B} T+\mathbf{h}_{\mathrm{thm}} \\
H_{\mathrm{SELM}} & =\Gamma(-\wp \mathcal{L})^{-1} \Lambda \\
\left\langle\mathbf{h}_{\mathrm{thm}}(s) \mathbf{h}_{\mathrm{thm}}^{T}(t)\right\rangle & =\left(2 k_{B} T\right) H_{\mathrm{SELM}} \delta(t-s) .
\end{aligned}
$$

The $\wp$ denotes a projection operator imposing constraints, such as incompressibility. The adjoint property $\Lambda=\Gamma^{\dagger}$ and symmetry of $\wp \mathcal{L}$ yields an operator $H_{\text {SELM }}$ which is symmetric. A more detailed discussion and derivation of the equations in this regime is given in Section 3.4.

2.5. Summary. This gives an overview of the SELM formalism and the associated stochastic differential equations. We remark that each of these regimes were motivated by a rather specific limit. Non-dimensional analysis of the equations can also be carried out and other limits considered to motivate working with such reduced equations. We discuss in more detail the derivation of the reduced equations in each regime in Section 3. We discuss how specific stochastic numerical methods can be developed for the SELM formalism in Section 4. We discuss applications and how the SELM formalism can be used in practice in Section 6 .

3. Derivations for the Stochastic Eulerian Lagrangian Method. We now discuss formal derivations to motivate the stochastic differential equations used in each of the physical regimes. For this purpose, we do not present the most general derivation of the equations. For brevity, we make simplifying assumptions when convenient.

In the initial formulation of SELM, the fluid-structure system is described by

$$
\rho \frac{d \mathbf{u}}{d t}=\mathcal{L} \mathbf{u}+\Lambda[\Upsilon(\mathbf{v}-\Gamma \mathbf{u})]+\lambda+\mathbf{f}_{\mathrm{thm}}
$$




$$
\begin{aligned}
m \frac{d \mathbf{v}}{d t} & =-\Upsilon(\mathbf{v}-\Gamma \mathbf{u})-\nabla_{\mathbf{X}} \Phi(\mathbf{X})+\zeta+\mathbf{F}_{\text {thm }} \\
\frac{d \mathbf{X}}{d t} & =\mathbf{v} .
\end{aligned}
$$

The notation and operators appearing in these equations has been discussed in detail in Section 2. For these equations, we focus primarily on the motivation for the stochastic driving fields used for the fluid-structure system.

For the thermal fluctuations of the system, we assume Gaussian random fields with mean zero and $\delta$-correlated in time. For such stochastic fields, the central challenge is to determine an appropriate covariance structure. For this purpose, we use the fluctuation-dissipation principle of statistical mechanics [54, 36]. For linear stochastic differential equations of the form

$$
d \mathbf{Z}_{t}=L \mathbf{Z}_{t} d t+Q d \mathbf{B}_{t}
$$

the fluctuation-dissipation principle can be expressed as

$$
G=Q Q^{T}=-(L C)-(L C)^{T} .
$$

This relates the equilibrium covariance structure $C$ of the system to the covariance structure $G$ of the stochastic driving field. The operator $L$ accounts for the dissipative dynamics of the system. For the equations $3.1-3.3$, the dissipative operators only appear in the momentum equations. This can be shown to have the consequence that there is no thermal forcing in the equation for $\mathbf{X}(t)$, this will also be confirmed in Section 5.1. To simplify the presentation, we do not represent explicitly the stochastic dynamics of the structure configuration $\mathbf{X}$.

For the fluid-structure system it is convenient to work with the stochastic driving fields by defining

$$
\mathbf{q}=\left[\rho^{-1} \mathbf{f}_{\mathrm{thm}}, m^{-1} \mathbf{F}_{\mathrm{thm}}\right]^{T} .
$$

The field $\mathbf{q}$ formally is given by $\mathbf{q}=Q d \mathbf{B}_{t} / d t$ and determined by the covariance structure $G=Q Q^{T}$. This covariance structure is determined by the fluctuationdissipation principle expressed in equation 3.5 with

$$
\begin{aligned}
L & =\left[\begin{array}{ll}
\rho^{-1}(\mathcal{L}-\Lambda \Upsilon \Gamma) & \rho^{-1} \Lambda \Upsilon \\
m^{-1} \Upsilon \Gamma & -m^{-1} \Upsilon
\end{array}\right] \\
C & =\left[\begin{array}{ll}
\rho^{-1} k_{B} T \mathcal{I} & 0 \\
0 & m^{-1} k_{B} T \mathcal{I}
\end{array}\right] .
\end{aligned}
$$

The $\mathcal{I}$ denotes the identity operator. The covariance $C$ was obtained by considering the fluctuations at equilibrium. The covariance $C$ is easily found since the GibbsBoltzmann distribution is a Gaussian with formal density $\Psi(\mathbf{u}, \mathbf{v})=\frac{1}{Z_{0}} \exp \left[-E / k_{B} T\right]$. The $Z_{0}$ is the normalization constant for $\Psi$. The energy is given by equation 2.4. For this purpose, we need only consider the energy $E$ in the case when $\Phi=0$. This gives the covariance structure

$$
G=\left(2 k_{B} T\right)\left[\begin{array}{ll}
-\rho^{-2}(\mathcal{L}-\Lambda \Upsilon \Gamma) & -m^{-1} \rho^{-1} \Lambda \Upsilon \\
-m^{-1} \rho^{-1} \Upsilon \Gamma & m^{-2} \Upsilon
\end{array}\right] .
$$

To obtain this result we use that $\Gamma=\Lambda^{\dagger}$ and $\Upsilon=\Upsilon^{\dagger}$. From the definition of $\mathbf{q}$, it is found the covariance of the stochastic driving fields of SELM are given by equations 2.6-2.8. This provides a description of the thermal fluctuations in the fluid-structure system. 
3.1. Regime I. It is convenient to reformulate the description of the fluidstructure system in terms of a field for the total momentum of the system associated with spatial location $\mathbf{x}$. For this purpose we define

$$
\mathbf{p}(\mathbf{x}, t)=\rho \mathbf{u}(\mathbf{x}, t)+\Lambda[m \mathbf{v}(t)](\mathbf{x}) .
$$

The operator $\Lambda$ is used to give the distribution in space of the momentum associated with the structures. Using this approach, the fluid-structure dynamics are described by

$$
\begin{aligned}
\frac{d \mathbf{p}}{d t} & =\mathcal{L} \mathbf{u}+\Lambda\left[-\nabla_{\mathbf{X}} \Phi(\mathbf{X})\right]+\left(\nabla_{\mathbf{X}} \Lambda[m \mathbf{v}]\right) \cdot \mathbf{v}+\lambda+\mathbf{g}_{\mathrm{thm}} \\
m \frac{d \mathbf{v}}{d t} & =-\Upsilon(\mathbf{v}-\Gamma \mathbf{u})-\nabla_{\mathbf{X}} \Phi(\mathbf{X})+\zeta+\mathbf{F}_{\mathrm{thm}} \\
\frac{d \mathbf{X}}{d t} & =\mathbf{v}
\end{aligned}
$$

where $\mathbf{u}=\rho^{-1}(\mathbf{p}-\Lambda[m \mathbf{v}])$ and $\mathbf{g}_{\mathrm{thm}}=\mathbf{f}_{\mathrm{thm}}+\Lambda\left[\mathbf{F}_{\mathrm{thm}}\right]$. The third term in the first equation arises from the dependence of $\Lambda$ on the configuration of the structures, $\Lambda[m \mathbf{v}(t)]=(\Lambda[X])[m \mathbf{v}(t)]$.

The thermal fluctuations are taken into account by two stochastic fields $\mathbf{g}_{\mathrm{thm}}$ and $\mathbf{F}_{\mathrm{thm}}$. The covariance of $\mathbf{g}_{\mathrm{thm}}$ is obtained from

$$
\begin{aligned}
\left\langle\mathbf{g}_{\mathrm{thm}} \mathbf{g}_{\mathrm{thm}}^{T}\right\rangle & =\left\langle\mathbf{f}_{\mathrm{thm}} \mathbf{f}_{\mathrm{thm}}^{T}\right\rangle+\left\langle\mathbf{f}_{\mathrm{thm}} \mathbf{F}_{\mathrm{thm}}^{T} \Lambda^{T}\right\rangle+\left\langle\Lambda \mathbf{F}_{\mathrm{thm}} \mathbf{f}_{\mathrm{thm}}^{T}\right\rangle+\left\langle\Lambda \mathbf{F}_{\mathrm{thm}} \mathbf{F}_{\mathrm{thm}}^{T} \Lambda^{T}\right\rangle \\
& =\left(2 k_{B} T\right)\left(-\mathcal{L}+\Lambda \Upsilon \Gamma-\Lambda \Upsilon \Lambda^{T}-\Lambda \Upsilon \Lambda^{T}+\Lambda \Upsilon \Lambda^{T}\right) \\
& =-\left(2 k_{B} T\right) \mathcal{L} .
\end{aligned}
$$

This makes use of the adjoint property of the coupling operators $\Lambda^{\dagger}=\Gamma$.

One particularly convenient feature of this reformulation is that the stochastic driving field $\mathbf{F}_{\text {thm }}$ and $\mathbf{g}_{\text {thm }}$ become independent. This can be seen as follows

$$
\begin{aligned}
\left\langle\mathbf{g}_{\mathrm{thm}} \mathbf{F}_{\mathrm{thm}}^{T}\right\rangle & =\left\langle\mathbf{f}_{\mathrm{thm}} \mathbf{F}_{\mathrm{thm}}^{T}\right\rangle+\left\langle\Lambda \mathbf{F}_{\mathrm{thm}} \mathbf{F}_{\mathrm{thm}}^{T}\right\rangle \\
& =\left(2 k_{B} T\right)(-\Lambda \Upsilon+\Lambda \Upsilon)=0 .
\end{aligned}
$$

This decoupling of the stochastic driving fields greatly reduces the computational effort to generate the fields with the required covariance structure. This shows the covariance structure of the stochastic driving fields of SELM are given by equations 2.132.15 .

3.2. Regime II. In many situations, inertial effects often play a relatively minor role in the structure dynamics as a consequence of the small mass of the structure relative to the displaced fluid or as a consequence of the large viscosity of the solvent fluid [31, 42]. We consider the regime in which $m \ll \rho \ell^{3}$, as discussed in Section 2.2. We shall derive formally reduced stochastic equations in the limit $m \rightarrow 0$.

For this purpose, we focus primarily on the dynamics of the velocity of the structures $\mathbf{v}(t)$. This can be expressed using the notation of Ito Stochastic Differential Equations [48] as

$$
d \mathbf{V}_{t}=-m^{-1} \Upsilon\left(\mathbf{V}_{t}-\Gamma \mathbf{u}+\Upsilon^{-1} \nabla_{\mathbf{X}} \Phi(\mathbf{X})\right) d t+m^{-1}\left(2 k_{B} T \Upsilon\right)^{1 / 2} d \mathbf{B}_{t}
$$

The $\mathbf{B}_{t}$ denotes throughout the standard Brownian motion on $\mathbb{R}^{N}$ [48]. To simplify the presentation, we consider the case when $\zeta=0$. We expect similar results to 
hold more generally. Treating the other degrees of freedom as fixed, we can solve equation 3.16 using Ito's Lemma [48]. The stationary behavior of this stochastic process can be expressed as

$$
\begin{aligned}
& \mathbf{V}_{t}=\boldsymbol{\mu}_{0}+\int_{-\infty}^{t} e^{-(t-s) m^{-1} \Upsilon} m^{-1}\left(2 k_{B} T \Upsilon\right)^{1 / 2} d \mathbf{B}_{s} \\
& \boldsymbol{\mu}_{0}=\Gamma \mathbf{u}-\Upsilon^{-1} \nabla_{\mathbf{X}} \Phi(\mathbf{X}) .
\end{aligned}
$$

As a result of the integrand being deterministic in the Ito Integral, the $\mathbf{V}_{t}$ is a Gaussian process. This has the consequence that the statistics of the process $\mathbf{V}_{t}$ are completely determined by its mean and covariance functions. The mean of the process is given at each time by

$$
\boldsymbol{\mu}(t)=\left\langle\mathbf{V}_{t}\right\rangle=\boldsymbol{\mu}_{0} .
$$

The covariance function can be computed using the Ito Isometry [48] to obtain

$$
\phi(|\tau|)=\left\langle\left(\mathbf{V}_{t+\tau}-\boldsymbol{\mu}_{0}\right)\left(\mathbf{V}_{t}-\boldsymbol{\mu}_{0}\right)^{T}\right\rangle=k_{B} T m^{-1} e^{-|\tau| m^{-1} \Upsilon} \mathcal{I} .
$$

In the limit $m \rightarrow 0$ this can be expressed as

$$
\phi(|t-s|)=2 k_{B} T \Upsilon^{-1}\left[\frac{1}{2} m^{-1} \Upsilon e^{-|t-s| m^{-1} \Upsilon}\right] \rightarrow 2 k_{B} T \Upsilon^{-1} \delta(t-s) .
$$

We have used formally $\frac{1}{2} \lambda e^{-\lambda|\tau|} \rightarrow \delta(\tau)$ as $\lambda \rightarrow \infty$. This suggests the following approximation for the velocity of the structures in equations 2.11 and 2.12 .

$$
\mathbf{v}(t) \rightarrow \Gamma \mathbf{u}-\Upsilon^{-1} \nabla_{\mathbf{X}} \Phi(\mathbf{X})+\left(2 k_{B} T \Upsilon^{-1}\right)^{1 / 2} \frac{d \mathbf{B}_{t}}{d t} .
$$

To approximate the term $\left(\nabla_{\mathbf{X}} \Lambda[m \mathbf{v}]\right) \cdot \mathbf{v}$ in the limit $m \rightarrow 0$ appearing in equation 2.10, a different approach is required. For this purpose, we consider the process $R_{t}=f\left(\mathbf{V}_{t}\right)=m \mathbf{V}_{t} \mathbf{V}_{t}^{T}$. By Ito's Lemma this satisfies the stochastic differential equation

$$
d R_{t}=\nabla f\left(\mathbf{V}_{t}\right) d \mathbf{V}_{t}+\frac{1}{2} d \mathbf{V}_{t}^{T} \nabla^{2} f\left(\mathbf{V}_{t}\right) d \mathbf{V}_{t}
$$

with the formal substitutions $d t d \mathbf{B}_{t}=0=d t d t, d \mathbf{B}_{t} d \mathbf{B}_{t}^{T}=\mathcal{I} d t$. To simplify the discussion we consider the case when $\Upsilon=\gamma \mathcal{I}$. We expect similar results can be obtained more generally. In this case

$$
\begin{aligned}
d R_{t} & =-2 m^{-1} \gamma\left(R_{t}-k_{B} T I-\frac{1}{2}\left(m \mathbf{V}_{t} \boldsymbol{\mu}_{0}^{T}+\boldsymbol{\mu}_{0}\left(m \mathbf{V}_{t}\right)^{T}\right)\right) d t \\
& +m^{-1}\left(2 k_{B} T \gamma\right)^{1 / 2}\left(m \mathbf{V}_{t} d \mathbf{B}_{t}^{T}+d \mathbf{B}_{t}\left(m \mathbf{V}_{t}\right)^{T}\right) .
\end{aligned}
$$

From the form of the energy given by equation 2.4, the structure momentum $m \mathbf{V}_{t}$ has equilibrium distribution $\Psi(m \mathbf{V})=\exp \left[-(m \mathbf{V})^{2} / 2 m k_{B} T\right]$. This gives a Gaussian with mean and variance

$$
\begin{aligned}
\left\langle m \mathbf{V}_{t}\right\rangle & =0 \\
\left\langle\left(m \mathbf{V}_{t}\right)\left(m \mathbf{V}_{t}\right)^{T}\right\rangle & =m k_{B} T \mathcal{I} .
\end{aligned}
$$


By reasoning similar to the arguments used to approximate $\mathbf{V}_{t}$, this suggests the terms involving $m \mathbf{V}_{t}$ do not make a contribution in the $m \rightarrow 0$ limit. This suggests to leading order we have

$$
d R_{t}=-2 m^{-1} \gamma\left(R_{t}-k_{B} T \mathcal{I}\right) d t .
$$

This suggests the approximation in the limit $m \rightarrow 0$

$$
R_{t}=m \mathbf{V}_{t} \mathbf{V}_{t}^{T} \rightarrow k_{B} T \mathcal{I} .
$$

By substituting this result for $\mathbf{v}$ in equation 2.10, we have

$$
\left(\nabla_{\mathbf{X}} \Lambda[m \mathbf{v}]\right) \cdot \mathbf{v} \rightarrow \operatorname{Tr}\left[\nabla_{\mathbf{X}} \Lambda\right]\left(k_{B} T\right)=\left(\nabla_{\mathbf{X}} \cdot \Lambda\right)\left(k_{B} T\right) .
$$

This establishes formally the reduced SELM description when the mass of the structures becomes negligible. It should be mentioned these approximations can be established more rigorously using a perturbation analysis of the Kolomogorov Equations associated with the stochastic processes [35, 25]. It should also be mentioned that other limits can be considered in which additional drift and stochastic terms arise in the reduced momentum equations. This will be the focus of another paper.

3.3. Regime III. The SELM stochastic equations can be further reduced if the effective viscous interactions between the structure and fluid are assumed to become very strong. This corresponds to approximating the reduced stochastic equations of Regime II in the formal limit $\Upsilon \rightarrow \infty$. By this notation, we mean that all eigenvalues of the symmetric operator $\Upsilon$ uniformly tend to infinity. In this formal limit the terms involving $\Upsilon^{-1}$ are expected to no longer make a contribution to the dynamics. This motivates the reduced stochastic equations 2.21- 2.23.

3.4. Regime IV. The description of the fluid-structure system can be further simplified by considering for the fluid the viscous limit in which $\mu \rightarrow \infty$. In this regime the fluid adopts a quasi-steady-state behavior with respect to the configuration of the structures and the forces acting on the fluid. In this limit only the structure dynamics remain. By approximating $\mathbf{u}(t)$ using arguments similar to those used in Regime II for approximating $\mathbf{V}_{t}$, we can derive the reduced stochastic equations 2.24- 2.26.

4. Computational Methodology. We now discuss briefly numerical methods for the SELM formalism. For concreteness we consider the specific case in which the fluid is Newtonian and incompressible. For now, the other operators of the SELM formalism will be treated rather generally. This case corresponds to the dissipative operator for the fluid

$$
\mathcal{L} \mathbf{u}=\mu \Delta \mathbf{u} .
$$

The $\Delta$ denotes the Laplacian $\Delta \mathbf{u}=\partial_{x x} \mathbf{u}+\partial_{y y} \mathbf{u}+\partial_{z z} \mathbf{u}$. The incompressibility of the fluid corresponds to the constraint

$$
\nabla \cdot \mathbf{u}=0
$$

This is imposed by the Lagrange multiplier $\lambda$. By the Hodge Decomposition, $\lambda$ is given by the gradient of a function $p$ with $\lambda=-\nabla p$. The $p$ can be interpreted as the local pressure of the fluid.

A variety of methods could be used in practice to discretize the SELM formalism, such as Finite Difference Methods, Spectral Methods, and Finite Element Methods $[29,60,59]$. We present here discretizations based on Finite Difference Methods. 
4.1. Numerical Semi-Discretizations for Incompressible Newtonian Fluid. The Laplacian will be approximated by central differences on a uniform periodic lattice by

$$
[L \mathbf{u}]_{\mathbf{m}}=\sum_{j=1}^{3} \frac{\mathbf{u}_{\mathbf{m}+\mathbf{e}_{j}}-2 \mathbf{u}_{\mathbf{m}}+\mathbf{u}_{\mathbf{m}-\mathbf{e}_{j}}}{\Delta x^{2}} .
$$

The $\mathbf{m}=\left(m_{1}, m_{2}, m_{3}\right)$ denotes the index of the lattice site. The $\mathbf{e}_{j}$ denotes the standard basis vector in three dimensions. The incompressibility of the fluid will be approximated by imposing the constraint

$$
[D \cdot \mathbf{u}]_{\mathbf{m}}=\sum_{j=1}^{3} \frac{\mathbf{u}_{\mathbf{m}+\mathbf{e}_{j}}^{j}-\mathbf{u}_{\mathbf{m}-\mathbf{e}_{j}}^{j}}{2 \Delta x} .
$$

The superscripts denote the vector component. In practice, this will be imposed by computing the projection of a vector $\mathbf{u}^{*}$ to the sub-space $\left\{\mathbf{u} \in \mathbb{R}^{3 N} \mid D \cdot \mathbf{u}=0\right\}$, where $N$ is the total number of lattice sites. We denote this projection operation by

$$
\mathbf{u}=\wp \mathbf{u}^{*} .
$$

The semi-discretized equations for SELM to be used in practice are

$$
\begin{aligned}
\frac{d \mathbf{p}}{d t} & =L \mathbf{u}+\Lambda\left[-\nabla_{\mathbf{X}} \Phi\right]+\left(\nabla_{\mathbf{X}} \Lambda[m \mathbf{v}]\right) \cdot \mathbf{v}+\lambda+\mathbf{g}_{\mathrm{thm}} \\
\frac{d \mathbf{v}}{d t} & =-\Upsilon[\mathbf{v}-\Gamma \mathbf{u}]+\mathbf{F}_{\mathrm{thm}} \\
\frac{d \mathbf{X}}{d t} & =\mathbf{v} .
\end{aligned}
$$

The component $\mathbf{u}_{\mathbf{m}}=\rho^{-1}\left(\mathbf{p}_{\mathbf{m}}-\Lambda[m \mathbf{v}]_{\mathbf{m}}\right)$. Each of the operators now appearing are understood to be discretized. We discuss specific discretizations for $\Gamma$ and $\Lambda$ in Section 6 . To obtain the Lagrange multiplier $\lambda$ which imposes incompressibility we use the projection operator and

$$
\lambda=-(\mathcal{I}-\wp)\left(L \mathbf{u}+\Upsilon[\mathbf{v}-\Gamma \mathbf{u}]+\mathbf{f}_{\mathrm{thm}}\right)
$$

In this expression, we let $\mathbf{f}_{\mathrm{thm}}=\mathrm{g}_{\mathrm{thm}}-\Lambda\left[\mathbf{F}_{\mathrm{thm}}\right]$ for the particular realized values of the fields $\mathbf{g}_{\text {thm }}$ and $\mathbf{F}_{\text {thm }}$.

We remark that in fact the semi-discretized equations of the SELM formalism in this regime can also be given in terms of $\mathbf{u}$ directly, which may provide a simpler approach in practice. The identity $\mathbf{f}_{\mathrm{thm}}=\mathrm{g}_{\mathrm{thm}}-\Lambda\left[\mathbf{F}_{\mathrm{thm}}\right]$ could be used to efficiently generate the required stochastic driving fields in the equations for $\mathbf{u}$. We present the reformulation here, since it more directly suggests the semi-discretized equations to be used for the reduced stochastic equations.

For this semi-discretization, we consider a total energy for the system given by

$$
E[\mathbf{u}, \mathbf{v}, \mathbf{X}]=\frac{\rho}{2} \sum_{\mathbf{m}}\left|\mathbf{u}\left(\mathbf{x}_{\mathbf{m}}\right)\right|^{2} \Delta \mathbf{x}_{\mathbf{m}}^{3}+\frac{m}{2}|\mathbf{v}|^{2}+\Phi[\mathbf{X}] .
$$

This is useful in formulating an adjoint condition 2.5 for the semi-discretized system. This can be derived by considering the requirements on the coupling operators $\Gamma$ 
and $\Lambda$ which ensure the energy is conserved when $\Upsilon \rightarrow \infty$ in the inviscid and zero temperature limit.

To obtain appropriate behaviors for the thermal fluctuations, it is important to develop stochastic driving fields which are tailored to the specific semi-discretizations used in the numerical methods. Once the stochastic driving fields are determined, which is the subject of the next section, the equations can be integrated in time using traditional methods for SDEs, such as the Euler-Maruyama Method or a Stochastic Runge-Kutta Method [34]. More sophisticated integrators in time can also be developed to cope with sources of stiffness, but are beyond the scope of this paper [6]. For each of the reduced equations, similar semi-discretizations can be developed as the one presented above.

4.2. Stochastic Driving Fields for Semi-Discretizations. To obtain behaviors consistent with statistical mechanics, it is important stochastic driving fields be used which are tailored to the specific numerical discretization employed [6, 21, 5]. To ensure consistency with statistical mechanics, we will again use the fluctuationdissipation principle but now apply it to the semi-discretized equations. For each regime, we then discuss the important issues arising in practice concerning the efficient generation of these stochastic driving fields.

4.3. Regime I. To obtain the covariance structure for this regime, we apply the fluctuation-dissipation principle as expressed in equation 3.5 to the semi-discretized equations 4.6- 4.8. This gives the covariance

$$
G=-2 L C=\left(2 k_{B} T\right)\left[\begin{array}{lll}
-\rho^{-2} \Delta x^{-3} L & 0 & 0 \\
0 & m^{-2} \Upsilon & 0 \\
0 & 0 & 0
\end{array}\right]
$$

The factor of $\Delta x^{-3}$ arises from the form of the energy for the discretized system which gives covariance for the equilibrium fluctuations of the total momentum $\rho^{-1} \Delta x^{-3} k_{B} T$, see equation 4.10. In practice, achieving the covariance associated with the dissipative operator of the fluid $L$ is typically the most challenging to generate efficiently. This arises from the large number $N$ of lattice sites in the discretization.

One approach is to determine a factor $Q$ such that the block $G_{\mathbf{p}, \mathbf{p}}=Q Q^{T}$, subscripts indicate block entry of the matrix. The required random field with covariance $G_{\mathbf{p}, \mathbf{p}}$ is then given by $\mathbf{g}=Q \boldsymbol{\xi}$, where $\boldsymbol{\xi}$ is the uncorrelated Gaussian field with the covariance structure $\mathcal{I}$. For the discretization used on the uniform periodic mesh, the matrices $L$ and $C$ are cyclic [58]. This has the important consequence that they are both diagonalizable in the discrete Fourier basis of the lattice. As a result, the field $\mathbf{f}_{\text {thm }}$ can be generated using the Fast Fourier Transform (FFT) with at most $O(N \log (N))$ computational steps. In fact, in this special case of the discretization, "random fluxes" at the cell faces can be used to generate the field in $O(N)$ computational steps [5]. Other approaches can be used to generate the random fields on non-periodic meshes and on multi-level meshes, see [4, 5].

4.4. Regime II. The covariance structure can be found using an approach similar to the one presented in Section 4.3. This gives

$$
G=\left(2 k_{B} T\right)\left[\begin{array}{ll}
-\rho^{-2} \Delta x^{-3} L & 0 \\
0 & \Upsilon^{-1}
\end{array}\right] .
$$

By factoring the covariance matrix in the Fourier basis, the field can be generated using FFTs in at most $O(N \log (N))$ computational steps. 
4.5. Regime III. The covariance structure can be found using an approach similar to the one presented in Section 4.3. This gives

$$
G=\left(2 k_{B} T\right)\left[\begin{array}{ll}
-\rho^{-2} \Delta x^{-3} L & 0 \\
0 & 0
\end{array}\right] .
$$

By factoring the covariance matrix in the Fourier basis, the field can be generated using FFT in at most $O(N \log (N))$ computational steps.

4.6. Regime IV. This regime differs from the others since the fluid momentum and structure momentum are both no longer represented explicitly. Spontaneous changes in the momentum of the system were the primary source of fluctuations in the configuration of the structures in the other regimes. While the momentum is no longer represented explicitly, we can none-the-less use a discretization of the momentum equations to generate efficiently the random fields required in the overdamped dynamics. This is done by expressing the covariance of the stochastic driving field as

$$
G=\left(2 k_{B} T\right) H_{\mathrm{SELM}}=\left(2 k_{B} T\right)\left(\Gamma \wp(-L)^{-1} \wp^{T} \Gamma^{T}\right) .
$$

This makes use of $\Lambda=\Gamma^{T}$ and properties of the specific discretized operators $L$ and $\wp$. In particular, commutativity $\wp L=L \wp$ and the projection operator properties $\wp^{2}=\wp, \wp=\wp^{T}$. Let $U$ be a factor so that $U U^{T}=-L^{-1}$. Using this factor we can express the covariance as

$$
G=\left(\sqrt{2 k_{B} T} \Gamma \wp U\right)\left(\sqrt{2 k_{B} T} \Gamma \wp U\right)^{T} .
$$

From this expression a matrix square-root of $G$ is readily obtained, $Q=\sqrt{2 k_{B} T} \Gamma \wp U$.

We remark this is different than the Cholesky factor obtained for $G$ which is required to be lower triangular $[61,58]$. Obtaining such a factor by Cholesky factorization would cost $O\left(M^{3}\right)$, where $M$ is the number of structure degrees of freedom. For the current discretization considered, the operators $L$ and $\wp$ are diagonalizable in Fourier space. This has the consequence that the action of the operators $U$ and $\wp$ can be computed using FFTs with a cost of $O(N \log (N))$. The $N$ is the number of lattice sites used to discretize $L$. The stochastic driving field is computed from $\mathbf{h}=Q \boldsymbol{\xi}$. This allows for the stochastic driving field to be generated in $O(N \log (N)+M)$ computational steps, assuming the action $\Lambda$ can be compute in $O(M)$ steps. This is in contrast to using the often non-sparse matrix arising from Cholesky factorization which generates the stochastic field with a cost of $O\left(M^{2}\right)$. We remark that this approach shares some similarities with the method proposed in $[7,56]$. Other methods based on splittings or multigrid can also be utilized to efficiently generate stochastic fields with this required covariance structure, see $[4,5]$.

5. Equilibrium Statistical Mechanics of SELM Dynamics. We now discuss how the SELM formalism and the presented numerical methods capture the equilibrium statistical mechanics of the fluid-structure system. This is done through an analysis of the invariant probability distribution of the stochastic dynamics. For the fluid-structure systems considered, the appropriate probability distribution is given by the Gibbs-Boltzmann distribution

$$
\Psi_{\mathrm{GB}}(\mathbf{z})=\frac{1}{Z} \exp \left[-E(\mathbf{z}) / k_{B} T\right] .
$$


The $\mathbf{z}$ is the state of the system, $E$ is the energy, $k_{B}$ is Boltzmann's constant, $T$ is the system temperature, and $Z$ is a normalization constant for the distribution [54]. We show this Gibbs-Boltzmann distribution is the equilibrium distribution of both the full stochastic dynamics and the reduced stochastic dynamics in each physical regime.

We present here both a verification of the invariance of the Gibbs-Boltzmann distribution for the general formalism and for numerical discretizations of the formalism. The verification is rather formal for the undiscretized formalism given technical issues which would need to be addressed for such an infinite dimensional dynamical system. However, the verification is rigorous for the semi-discretization of the formalism, which yields a finite dimensional dynamical system. The latter is likely the most relevant case in practice. Given the nearly identical calculations involved in the verification for the general formalism and its semi-discretizations, we use a notation in which the key differences between the two cases primarily arise in the definition of the energy. In particular, the energy is understood to be given by equation 2.4 when considering the general SELM formalism and equation 4.10 when considering semi-discretizations.

5.1. Regime I. The stochastic dynamics given by equations $2.10-2.12$ is a change-of-variable of the full stochastic dynamics of the SELM formalism given by equations $2.1-2.3$. Thus verifying the invariance using the reformulated description is also applicable to equations $2.1-2.3$ and vice versa. To verify the invariance in the other regimes, it is convenient to work with the reformulated description given for Regime I. The energy associated with the reformulated description is given by

$$
E[\mathbf{p}, \mathbf{v}, \mathbf{X}]=\frac{1}{2 \rho} \int_{\Omega}|\mathbf{p}(\mathbf{y})-\Lambda[m \mathbf{v}](\mathbf{y})|^{2} d \mathbf{y}+\frac{m}{2}|\mathbf{v}|^{2}+\Phi[\mathbf{X}]
$$

The energy associated with the semi-discretization is

$$
E[\mathbf{p}, \mathbf{v}, \mathbf{X}]=\frac{1}{2 \rho} \sum_{\mathbf{m}}\left|\mathbf{p}\left(\mathbf{x}_{\mathbf{m}}\right)-\Lambda[m \mathbf{v}]_{\mathbf{m}}\right|^{2} \Delta \mathbf{x}_{\mathbf{m}}^{3}+\frac{m}{2}|\mathbf{v}|^{2}+\Phi[\mathbf{X}] .
$$

The probability density $\Psi(\mathbf{p}, \mathbf{v}, \mathbf{X}, t)$ for the current state of the system under the SELM dynamics is governed by the Fokker-Planck equation

$$
\frac{\partial \Psi}{\partial t}=-\nabla \cdot \mathbf{J}
$$

with probability flux

$$
\mathbf{J}=\left[\begin{array}{l}
\mathcal{L}+\Lambda+\nabla_{\mathbf{X}} \Lambda \cdot \mathbf{v}+\lambda \\
-\Upsilon-\nabla_{\mathbf{X}} \Phi+\zeta \\
\mathbf{v}
\end{array}\right] \Psi-\frac{1}{2}(\nabla \cdot G) \Psi-\frac{1}{2} G \nabla \Psi .
$$

The covariance operator $G$ is associated with the Gaussian field $\mathbf{g}=\left[\mathbf{g}_{\mathrm{thm}}, \mathbf{F}_{\mathrm{thm}}, 0\right]^{T}$ by $\left\langle\mathbf{g}(s) \mathbf{g}^{T}(t)\right\rangle=G \delta(t-s)$. In this regime, $G$ is given by equation 2.13 or 4.11 . In the notation $[\nabla \cdot G(\mathbf{z})]_{i}=\partial_{z_{j}} G_{i j}(\mathbf{z})$ with the summation convention for repeated indices. To simplify the notation we have suppressed denoting the specific functions on which each of the operators act, see equations $2.10-2.12$ for these details.

The requirement that the Gibbs-Boltzmann distribution $\Psi_{\mathrm{GB}}$ given by equation 5.1 be invariant under the stochastic dynamics is equivalent to the distribution yielding $\nabla \cdot \mathbf{J}=0$. We find it convenient to group terms and express this condition as

$$
\nabla \cdot \mathbf{J}=A_{1}+A_{2}+\nabla \cdot \mathbf{A}_{3}+\nabla \cdot \mathbf{A}_{4}=0
$$


where

$$
\begin{aligned}
& A_{1}=\left[\left(\Lambda+\nabla_{\mathbf{X}} \Lambda \cdot \mathbf{v}+\lambda_{1}\right) \cdot \nabla_{\mathbf{p}} E+\left(-\nabla_{\mathbf{X}} \Phi+\zeta_{1}\right) \cdot \nabla_{\mathbf{v}} E+(\mathbf{v}) \cdot \nabla_{\mathbf{X}} E\right]\left(-k_{B} T\right)^{-1} \Psi_{\mathrm{GB}} \\
& A_{2}= {\left[\nabla_{\mathbf{p}} \cdot\left(\Lambda+\nabla_{\mathbf{X}} \Lambda \cdot \mathbf{v}+\lambda_{1}\right)+\nabla_{\mathbf{v}} \cdot\left(-\nabla_{\mathbf{X}} \Phi+\zeta_{2}\right)+\nabla_{\mathbf{X}} \cdot(\mathbf{v})\right] \Psi_{\mathrm{GB}} } \\
& \mathbf{A}_{3}=-\frac{1}{2}(\nabla \cdot G) \Psi_{\mathrm{GB}} \\
& \mathbf{A}_{4}=\left[\begin{array}{l}
\mathcal{L} \mathbf{u}+\lambda_{2}+\left[G_{\mathbf{p}} \nabla_{\mathbf{p}} E+G_{\mathbf{p v}} \nabla_{\mathbf{v}} E+G_{\mathbf{p} \mathbf{X}} \nabla_{\mathbf{X}} E\right]\left(2 k_{B} T\right)^{-1} \\
-\Upsilon+\zeta_{2}+\left[G_{\mathbf{v p}} \nabla_{\mathbf{p}} E+G_{\mathbf{v v}} \nabla_{\mathbf{v}} E+G_{\mathbf{v} \mathbf{X}} \nabla_{\mathbf{X}} E\right]\left(2 k_{B} T\right)^{-1} \\
{\left[G_{\mathbf{X}} \nabla_{\mathbf{p}} E+G_{\mathbf{X} \mathbf{v}} \nabla_{\mathbf{v}} E+G_{\mathbf{X} \mathbf{x}} \nabla_{\mathbf{X}} E\right]\left(2 k_{B} T\right)^{-1}}
\end{array}\right] \Psi_{\mathrm{GB}} .
\end{aligned}
$$

We assume here that the Lagrange multipliers can be split $\lambda=\lambda_{1}+\lambda_{2}$ and $\zeta=\zeta_{1}+\zeta_{2}$ to impose the constraints by considering in isolation different terms contributing to the dynamics, see equation 5.7. This is always possible for linear constraints. The block entries of the covariance operator $G$ are denoted by $G_{i, j}$ with $i, j \in\{\mathbf{p}, \mathbf{v}, \mathbf{X}\}$. For the energy of the discretized system given by equation 2.4 we have

$$
\begin{aligned}
\nabla_{\mathbf{p}_{\mathbf{n}}} E & =\mathbf{u}\left(\mathbf{x}_{\mathbf{n}}\right) \Delta x_{\mathbf{n}}^{3} \\
\nabla_{\mathbf{v}_{q}} E & =\sum_{\mathbf{m}} \mathbf{u}\left(\mathbf{x}_{\mathbf{m}}\right) \cdot\left(-\nabla_{\mathbf{v}_{q}} \Lambda[m \mathbf{v}]_{\mathbf{m}}\right) \Delta x_{\mathbf{m}}^{3}+m \mathbf{v}_{q} \\
\nabla_{\mathbf{X}_{q}} E & =\sum_{\mathbf{m}} \mathbf{u}\left(\mathbf{x}_{\mathbf{m}}\right) \cdot\left(-\nabla_{\mathbf{x}_{q}} \Lambda[m \mathbf{v}]_{\mathbf{m}}\right) \Delta x_{\mathbf{m}}^{3}+\nabla_{\mathbf{X}_{q}} \Phi
\end{aligned}
$$

where $\mathbf{u}=\rho^{-1}(\mathbf{p}-\Lambda[m \mathbf{v}])$. Similar expressions for the energy of the undiscretized formalism can be obtained by using the calculus of variations [26].

We now consider $\nabla \cdot \mathbf{J}$ and each term $A_{1}, A_{2}, \mathbf{A}_{3}, \mathbf{A}_{4}$. The term $A_{1}$ can be shown to be the time derivative of the energy $A_{1}=d E / d t$ when considering only a subset of the contributions to the dynamics. Thus, conservation of the energy under this restricted dynamics would result in $A_{1}$ being zero. For the SELM formalism, we find by direct substitution of the gradients of $E$ given by equations 5.8- 5.10 into equation 5.7 that $A_{1}=0$. When there are constraints, it is important to consider only admissible states $(\mathbf{p}, \mathbf{v}, \mathbf{X})$. This shows in the inviscid and zero temperature limit of SELM the resulting dynamics are non-dissipative. This property imposes constraints on the coupling operators and can be viewed as a further motivation for the adjoint conditions imposed in equation 2.5.

The term $A_{2}$ gives the compressibility of the phase-space flow generated by the non-dissipative dynamics of the SELM formalism. The flow is generated by the vector field $\left(\Lambda+\nabla_{\mathbf{X}} \Lambda \cdot \mathbf{v}+\lambda_{1},-\nabla_{\mathbf{X}} \Phi+\zeta_{1}, \mathbf{v}\right)$ on the phase-space $(\mathbf{p}, \mathbf{v}, \mathbf{X})$. When this term is non-zero there are important implications for the Liouville Theorem and statistical mechanics of the system [62]. For the current regime, we have $A_{2}=0$ since in the divergence each component of the vector field is seen to be independent of the variable on which the derivative is computed. This shows in the inviscid and zero temperature limit of SELM, the phase-space flow is incompressible. For the reduced SELM descriptions, we shall see this is not always the case.

The term $\mathbf{A}_{3}$ corresponds to fluxes arising from multiplicative features of the stochastic driving fields. When the covariance $G$ has a dependence on the current state of the system, this can result in possible changes in the amplitude and correlations in the fluctuations. These changes can yield asymmetries in the stochastic dynamics which manifest as a net probability flux. In the SELM formalism it is found that in 
the divergence of $G$ each contributing entry is independent of the variable on which the derivative is being computed. This shows for the SELM dynamics there is no such probability fluxes, $\mathbf{A}_{3}=0$.

The last term $\mathbf{A}_{4}$ accounts for the fluxes arising from the primarily dissipative dynamics and the stochastic driving fields. This term is calculated by substituting the gradients of the energy given by equation 5.8-5.10 and using the choice of covariance structure given by equations 2.13 or 4.11 . By direct substitution this term is found to be zero, $\mathbf{A}_{4}=0$.

This shows the invariance of the Gibbs-Boltzmann distribution under the SELM dynamics. This provides a rather strong validation of the stochastic driving fields introduced for the SELM formalism. This shows the SELM stochastic dynamics are consist with equilibrium statistical mechanics [54].

5.2. Regime II. For the reduced stochastic dynamics given by equations 2.10 2.12 , the probability density $\Psi(\mathbf{p}, \mathbf{X}, t)$ satisfies the Fokker-Planck equation with the probability flux

$$
\mathbf{J}=\left[\begin{array}{l}
\rho^{-1} \mathcal{L}+\Lambda+\left(\nabla_{\mathbf{X}} \cdot \Lambda\right) k_{B} T+\lambda \\
\rho^{-1} \Gamma+\Upsilon^{-1}+\zeta
\end{array}\right] \Psi_{\mathrm{GB}}-\frac{1}{2}(\nabla \cdot G) \Psi_{\mathrm{GB}}-\frac{1}{2} G \nabla \Psi_{\mathrm{GB}}
$$

The $G$ denotes the covariance operator for the stochastic driving fields given by equation 4.12. The invariance of the Gibbs-Boltzmann distribution requires

$$
\begin{aligned}
\nabla \cdot \mathbf{J} & =A_{1}+A_{2}+\nabla \cdot \mathbf{A}_{3}+\nabla \cdot \mathbf{A}_{4}=0 \\
A_{1} & =\left[\left(\Lambda+\left(\nabla_{\mathbf{X}} \cdot \Lambda\right) k_{B} T+\lambda_{1}\right) \cdot \nabla_{\mathbf{p}} E+\left(\rho^{-1} \Gamma+\zeta_{1}\right) \cdot \nabla_{\mathbf{X}} E\right]\left(-k_{B} T\right)^{-1} \Psi_{\mathrm{GB}} \\
A_{2} & =\left[\nabla_{\mathbf{p}} \cdot\left(\Lambda+\left(\nabla_{\mathbf{X}} \cdot \Lambda\right) k_{B} T+\lambda_{1}\right)+\nabla_{\mathbf{X}} \cdot\left(\rho^{-1} \Gamma+\zeta_{1}\right)\right] \Psi_{\mathrm{GB}} \\
\mathbf{A}_{3} & =-\frac{1}{2}(\nabla \cdot G) \Psi_{\mathrm{GB}} \\
\mathbf{A}_{4} & =\left[\begin{array}{l}
\left(\rho^{-1} \mathcal{L}+\lambda_{2}\right)+\left[G_{\mathbf{p} \mathbf{p}} \nabla_{\mathbf{p}} E+G_{\mathbf{p} \mathbf{X}} \nabla_{\mathbf{X}} E\right]\left(2 k_{B} T\right)^{-1} \\
\left(\Upsilon^{-1}+\zeta_{2}\right)+\left[G_{\mathbf{X}} \nabla_{\mathbf{p}} E+G_{\mathbf{X X}} \nabla_{\mathbf{X}} E\right]\left(2 k_{B} T\right)^{-1}
\end{array}\right] \Psi_{\mathrm{GB}} .
\end{aligned}
$$

To simplify the notation we have suppressed explicitly denoting the functions on which the operators act, which can be inferred from equation 2.16-2.17. In the current regime $m=0$ and the energy given by equation 4.10 has gradients given by

$$
\begin{aligned}
\nabla_{\mathbf{p}_{\mathbf{n}}} E & =\mathbf{u}_{\mathbf{n}} \Delta x_{\mathbf{n}}^{3} \\
\nabla_{\mathbf{x}_{q}} E & =\nabla_{\mathbf{X}_{q}} \Phi .
\end{aligned}
$$

Similar expressions can be obtained for the undiscretized formalism using the calculus of variations [26].

We now consider $\nabla \cdot \mathbf{J}$ and $A_{1}, A_{2}, \mathbf{A}_{3}, \mathbf{A}_{4}$. The terms have a similar interpretation as in Section 5.1. The $A_{1}$ term can be interpreted as the time derivative of the energy $A_{1}=d E / d t$ when considering only a subset of the contributions to the dynamics. By direct substitution of the gradients given by equation 5.13-5.14, we find $A_{1}=$ $-\left(\left(\nabla_{\mathbf{X}} \cdot \Lambda\right) \cdot \nabla_{\mathbf{p}} E\right) \Psi_{\mathrm{GB}}$. This differs from the non-reduced equations in which this term was zero, see Section 5.1.

The term $A_{2}$ gives the compressibility of the flow generated by the vector field $\left(\Lambda+\left(\nabla_{\mathbf{X}} \cdot \Lambda\right) k_{B} T+\lambda_{1}, \rho^{-1} \Gamma+\zeta_{1}\right)$ on the phase-space $(\mathbf{p}, \mathbf{X})$. For the reduced equations, the phase-space flow has compressibility given by $A_{2}=\left(\rho^{-1} \nabla_{\mathbf{X}} \cdot \Gamma\right) \Psi_{\mathrm{GB}}$, 
which in general is no longer zero. However, we have that $A_{1}+A_{2}=0$. This follows from the form of the gradients given by equation 5.13- 5.14 and from the properties of $\Gamma$ and $\Lambda$. In particular, that the operators are linear and that they are adjoints $\Gamma=\Lambda^{\dagger}$ in the sense of equation 2.5.

The term $\mathbf{A}_{3}$ accounts for probability fluxes driven by multiplicative features of the stochastic driving fields. It is found this term is zero $\mathbf{A}_{3}=0$. This follows from the divergence in which each entry of $G$ is independent of the variable on which the derivative is applied. The term $\mathbf{A}_{4}$ accounts for fluxes arising from the dissipative contributions to the dynamics and the stochastic driving fields. By direct substitution of the gradients given in equation 5.13- 5.14, and the choice made for $G$ given in equation 4.12, we find this term is zero, $\mathbf{A}_{4}=0$. This establishes $\nabla \cdot \mathbf{J}=0$ and that the Gibbs-Boltzmann distribution is invariant for the SELM dynamics.

5.3. Regime III. We now discuss briefly the reduced stochastic dynamics given by equations $2.21-2.23$, in which $\Upsilon \rightarrow \infty$. In this regime, the probability flux is almost identical to equation 5.11, with any terms involving $\Upsilon^{-1}$ set to zero. With this substitution, it immediately follows that the Gibbs-Boltzmann distribution is invariant under the SELM dynamics.

5.4. Regime IV. In the over-damped regime in which the fluid is no longer explicitly represented, we have the reduced stochastic dynamics given by equations $2.24-$ 2.26. The probability density $\Psi(\mathbf{X}, t)$ for the current state of the system is governed by the Fokker-Planck equation with the probability flux

$$
\mathbf{J}=\left[H_{\mathrm{SELM}}\left[-\nabla_{\mathbf{X}} \Phi\right]+k_{B} T\left(\nabla_{\mathbf{X}} \cdot H_{\mathrm{SELM}}\right)\right] \Psi_{\mathrm{GB}}-\frac{1}{2}\left(\nabla_{\mathbf{X}} \cdot G\right) \Psi_{\mathrm{GB}}-\frac{1}{2} G \nabla_{\mathbf{X}} \Psi_{\mathrm{GB}} .
$$

In this regime, the covariance is given by $G=2 k_{B} T H_{\mathrm{SELM}}$, see Section 3.4. This gives $\nabla_{\mathbf{X}} \cdot G=2 k_{B} T \nabla_{\mathbf{X}} \cdot H_{\mathrm{SELM}}$ and $\frac{1}{2} G \nabla_{\mathbf{X}} \Psi_{\mathrm{GB}}=H_{\mathrm{SELM}}\left[-\nabla_{\mathbf{X}} \Phi\right] \Psi_{\mathrm{GB}}$. Substituting these expressions in equation 5.15, we find $\mathbf{J}=0$. This establishes for the over-damped regime of the SELM formalism that the Gibbs-Boltzmann distribution is invariant and satisfies detailed balance.

5.5. Summary. For the SELM formalism, we have demonstrated in each regime that the Gibbs-Boltzmann distribution is invariant. This shows the SELM formalism yields appropriate behaviors with respect to equilibrium statistical mechanics.

6. Applications. To demonstrate how the SELM formalism can be used in practice, we consider spherical particles which have translation and rotational degrees of freedom. We give specific operators representing the coupling of the particles and fluid. We compare this SELM formalism with classical results from fluid mechanics. We should mention that similar approaches for the SELM formalism can be applied much more generally to represent spatially extended structures, such as filaments, membranes, or even deformable bodies. The development of representations and specific coupling operators for these structures will be the focus of future work.

6.1. Particles with Rotational and Translational Degrees of Freedom. To describe particles which can exhibit translational and rotational motions, we use the degrees of freedom $\mathbf{X}_{\mathrm{cm}}$ for the center of mass and $\boldsymbol{\Theta}$ for the rotational configuration. To describe the full configuration of a particle, we define the composite vector $\mathbf{X}=\left(\mathbf{X}_{\mathrm{cm}}, \boldsymbol{\Theta}\right)$. To investigate how the coupling operators capture the hydrodynamics of the system, it is convenient to characterize the system in Regimes III and IV of 
Sections 2.3 and 2.4. This highlights central features of the coupling operators also relevant in the other regimes. Given the specific degrees of freedom of the particles, it is convenient to express equation 2.22 as

$$
\begin{aligned}
\frac{\partial \mathbf{X}_{\mathrm{cm}}}{\partial t} & =\Gamma_{0} \mathbf{u} \\
\frac{\partial \boldsymbol{\Theta}}{\partial t} & =\Gamma_{1} \mathbf{u} .
\end{aligned}
$$

The $\mathbf{u}=\rho^{-1} \mathbf{p}$. To represent the kinematics of such particles for a given state of the flow field of the fluid we use

$$
\begin{aligned}
& \Gamma_{0} \mathbf{u}=\sum_{\mathbf{m}}\left\langle\eta_{0}\left(\mathbf{y}_{\mathbf{m}}-\left(\mathbf{X}_{\mathrm{cm}}+\mathbf{z}\right)\right) \mathbf{u}_{\mathbf{m}}\right\rangle_{\tilde{\mathcal{S}},|\mathbf{z}|=R} \Delta x_{\mathbf{m}}^{3} \\
& \Gamma_{1} \mathbf{u}=\frac{3}{2 R^{2}} \sum_{\mathbf{m}}\left\langle\eta_{1}\left(\mathbf{y}_{\mathbf{m}}-\left(\mathbf{X}_{\mathrm{cm}}+\mathbf{z}\right)\right)\left(\mathbf{z} \times \mathbf{u}_{\mathbf{m}}\right)\right\rangle_{\tilde{\mathcal{S}},|\mathbf{z}|=R} \Delta x_{\mathbf{m}}^{3} .
\end{aligned}
$$

The angle brackets denote an average over the surface of the sphere which is given by the quadrature

$$
\langle f(\mathbf{z})\rangle_{\tilde{\mathcal{S}},|\mathbf{z}|=R}=\frac{1}{4 \pi R^{2}} \sum_{k} w_{k} f\left(\mathbf{z}_{k}\right)
$$

The $w_{k}$ denote the quadrature weights and the $\mathbf{z}_{k}$ denote the quadrature nodes. In practice, we use the Lebedev quadratures [39].

These SELM kinematics are in fact closely related to the exact kinematics of a passive spherical particle expressed using the Faxen Theorem of fluid dynamics [8]. The expression from the Faxen Theorem corresponds to the continuum limit of the above expressions and when the kernel functions are replaced by Dirac $\delta$-functions $[8$, $42,32]$.

For particles which actively exert forces on the fluid, we develop a coupling operator for the force by using the adjoint condition given by equation 2.5. In fact, this condition can be interpreted as requiring the fluid-structure coupling conserve the energy of the system in Regime III in the inviscid and zero temperature limit. This is seen formally by letting $\mathcal{L} \rightarrow 0$ and $T \rightarrow 0$ in equation 2.21 and computing what is required for $d E / d t=0$. Using this condition, we obtain the fluid coupling operator by considering directly the adjoint condition $\Lambda=\Gamma^{\dagger}$. For the specific coupling operators considered for the particles, the adjoint condition of the discretized system gives

$$
\begin{aligned}
& \Lambda_{0}\left(\mathbf{x}_{\mathbf{m}}\right)=\left(\left\langle\eta_{0}\left(\mathbf{x}_{\mathbf{m}}-\left(\mathbf{X}_{\mathrm{cm}}+\mathbf{z}\right)\right)\right\rangle_{\tilde{\mathcal{S}},|\mathbf{z}|=R}\right) \mathbf{F} \\
& \Lambda_{1}\left(\mathbf{x}_{\mathbf{m}}\right)=-\frac{3}{2 R^{2}}\left(\left\langle\mathbf{z} \eta_{1}\left(\mathbf{x}_{\mathbf{m}}-\left(\mathbf{X}_{\mathrm{cm}}+\mathbf{z}\right)\right)\right\rangle_{\tilde{\mathcal{S}},|\mathbf{z}|=R}\right) \times \mathbf{T} .
\end{aligned}
$$

The $\mathbf{F}=-\partial \Phi / \partial \mathbf{X}_{\mathrm{cm}}$ is the total force acting on the particle and $\mathbf{T}=-\partial \Phi / \partial \boldsymbol{\Theta}$ is the total torque acting on the particle. The adjoint condition holds exactly for the discretized system provided the same quadrature is used for both $\Gamma$ and $\Lambda$.

In the SELM formalism, the coupling operators $\Gamma$ and $\Lambda$ encapsulate the effective hydrodynamic coupling between the particles and fluid. To characterize how the presented SELM operators represent such hydrodynamic coupling in practice, we consider the interactions between two spherical particles. The coupling of two spherical particles has three modes of coupling: (i) translation-translation, (ii) rotation-rotation, 

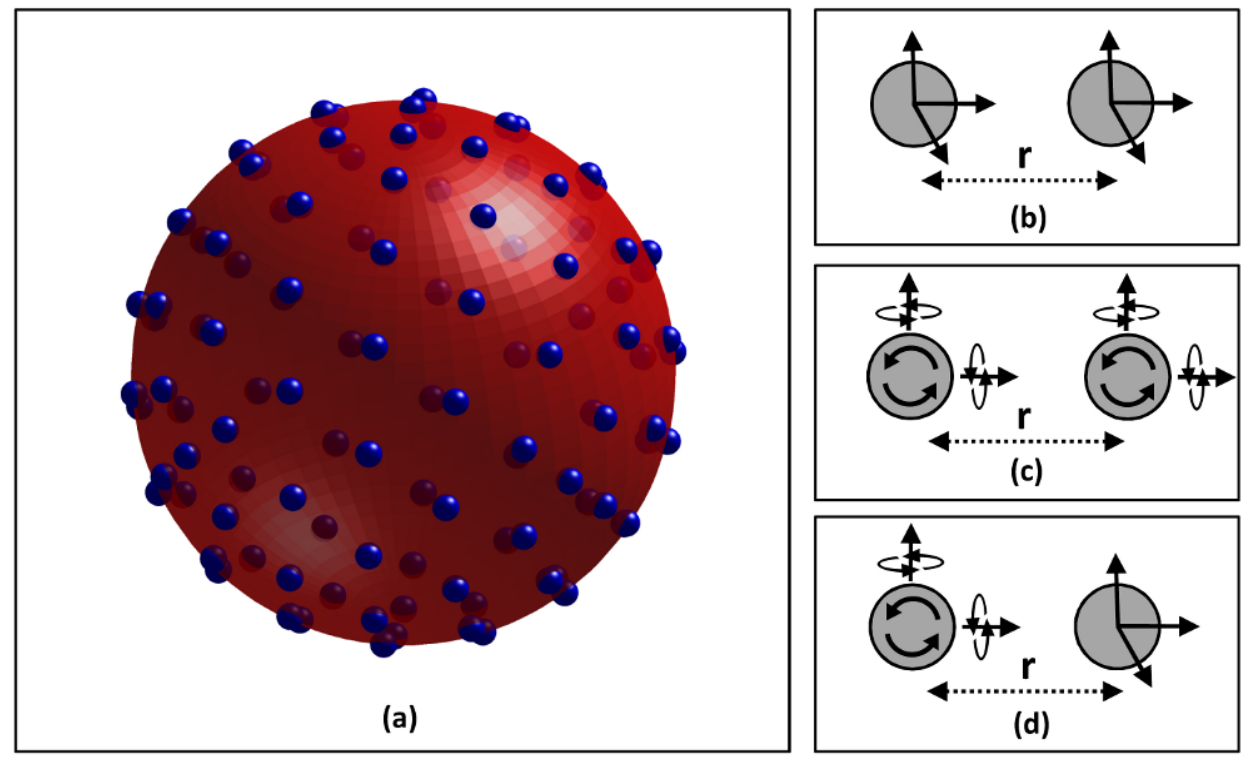

Fig. 6.1. Translational and Rotational Motions of Spherical Particles. On the left is shown the Lebedev quadrature nodes for $N=110$ which are used for computing averages on the surface of a sphere used in the SELM coupling operators. On the right is shown the three modalities of coupling for spherical particles. These are Translation-Translation (b), Rotation-Rotation (c), and Rotation-Translation (d).

and (iii) rotation-translation, see Figure 6.1. To characterize these coupling modes, we consider the effective hydrodynamic coupling tensor (mobility) which relates applied forces and torques to consequent motions of the particles. An effective coupling tensor can be determined rather naturally for the SELM formalism by considering Regime IV at zero temperature. In this regime, the effective coupling tensor arises in the dynamics

$$
\begin{aligned}
\frac{d}{d t}\left[\begin{array}{l}
\mathbf{X}_{\mathrm{cm}} \\
\mathbf{\Theta}
\end{array}\right] & =\left[\begin{array}{ll}
\tilde{H}_{\mathrm{TT}} & \tilde{H}_{\mathrm{TR}} \\
\tilde{H}_{\mathrm{RT}} & \tilde{H}_{\mathrm{RR}}
\end{array}\right]\left[\begin{array}{l}
\mathbf{F} \\
\mathbf{T}
\end{array}\right] \\
\tilde{H}_{\ell k} & =\Gamma_{\ell}(\wp L)^{-1} \Lambda_{k} .
\end{aligned}
$$

The tilde is used throughout to distinguish the tensor components associated with the SELM formalism from those arising in other theories from fluid mechanics. The subscripts $T, R$ indicates components related respectively to the translational and rotational degrees of freedom. In practice, the components of these tensors can be easily computed from an implementation of SELM by applying forces or torques which are set to $\mathbf{e}_{j}$ on only one particle and considering the $i^{\text {th }}$ component of the velocity or angular velocity of the other particle.

To compare the results of the proposed SELM coupling operators with classical results of fluid mechanics, we consider the following Rotne-Prager-Yamakawa tensors (RPY tensors) [55, 66, 53]

$$
H_{\mathrm{TT}}(\mathbf{r})=\left\{\begin{array}{ll}
\frac{1}{8 \pi \mu r}\left(1+\frac{2 a^{2}}{3 r^{2}}\right) \mathcal{I}+\frac{1}{8 \pi \mu r}\left(1-\frac{2 a^{2}}{r^{2}}\right) \hat{\mathbf{r}}^{T} \hat{\mathbf{r}}^{T}, & \text { for } r \geq 2 a \\
\frac{1}{16 \pi \mu a}\left(\frac{8}{3}-\frac{3 r}{4 a}\right) \mathcal{I}+\frac{r}{64 \pi \mu a^{2}} \hat{\mathbf{r}}^{T}, & \text { for } r<2 a
\end{array}\right\}
$$



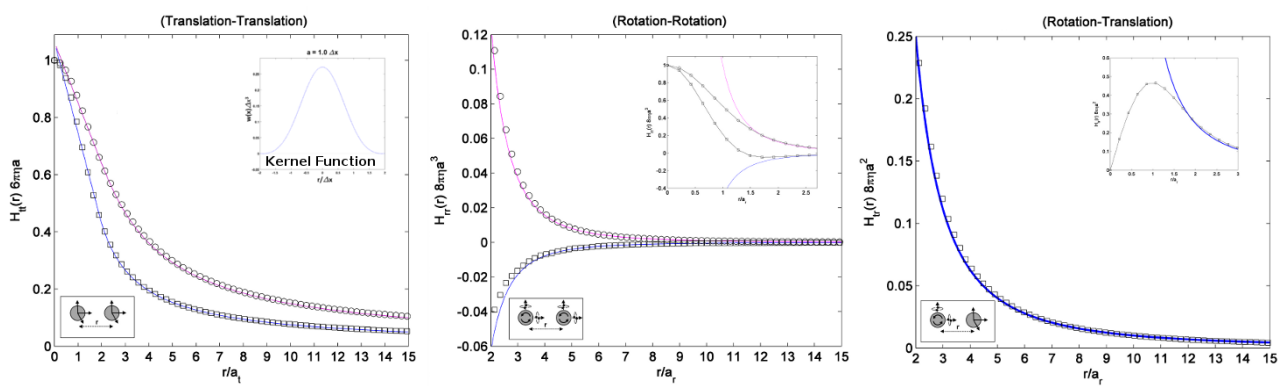

FIG. 6.2. Effective Hydrodynamic Coupling Tensors of the SELM Operators. The components of the effective hydrodynamic coupling tensor associated with the SELM operators are compared with the Rotne-Prager-Yamakawa Tensors of fluid mechanics. All components are scaled by the reference mobility $m_{t}=1 / 6 \pi \mu a_{t}$ for translation and $m_{r}=1 / 8 \pi \mu a_{r}^{3}$ for rotation. The kernel functions $\eta_{0}$ and $\eta_{1}$ were chosen to be the radial symmetric function shown as the inset on the left. The other insets show the near-field hydrodynamic interactions.

$$
\begin{aligned}
& H_{\mathrm{RR}}(\mathbf{r})=\frac{1}{16 \pi \mu r^{3}}\left(\mathcal{I}-3 \hat{\mathbf{r}} \hat{\mathbf{r}}^{T}\right) \\
& H_{\mathrm{TR}}(\mathbf{r})=\frac{1}{8 \pi \mu r^{2}} \hat{\mathbf{r}} \times .
\end{aligned}
$$

In this notation, $\hat{\mathbf{r}}=\mathbf{r} / r$ and we denote by $\hat{\mathbf{r}} \times$ the matrix which represents the action of the cross-product of a vector with $\hat{\mathbf{r}}$. The $a$ denotes the effective hydrodynamic radius of the particle. The RPY tensors capture far-field interactions and are expected to be accurate physically only when the particles are sufficiently separated. They are not designed to capture lubrication interactions or other near-field effects $[11,1,55$, $66]$.

For the proposed coupling operators, very good agreement is found with the RPY tensors for all of the different coupling modes, see Figure 6.2. This agreement is especially good for particles separated at least a distance of two radii. At closer distances the RPY tensors either reflect some type of regularization or they diverge. In the case of translation-translation coupling the SELM formalism agrees even in the nearfield with the RPY tensor [55, 66]. For the other cases, the RPY tensors diverge. In all cases, the SELM formalism provides as the separation distance becomes small a regularized tensor. The regularization can be interpreted as an interpolation between the two particle far-field interaction to the single particle response, see insets in Figure 6.2. For the rotation-translation coupling model, we see the operators have the important property for the single particle response that the rotation and translational motions are decoupled. This agrees with predictions from fluid mechanics for linear Stokes flow.

These results for the proposed operators demonstrate that the SELM formalism provides a practical approach for simulating hydrodynamically coupled spherical particles. We remark that the near-field artifacts are a by-product of the specific coupling operators utilized to approximate the fluid-structure interactions. For many practical systems, repulsive long-range interactions keep particles well-separated avoiding these near-field artifacts. It should be emphasized the SELM formalism is not limited to such cases, since a more accurate approach which captures near-field effects can be developed by using a different choice for the coupling operators.

While we have presented only one rather special application, the SELM formalism can be applied more broadly. This simply requires appropriate representations for the 
structures and a choice for the coupling operators. It is expected a wide variety of structures could be studied using this approach, including particles of non-spherical shape, filaments, membranes, and deformable bodies. The development of coupling operators for these systems will be the focus of future work.

7. Conclusions. An approach for fluid-structure interactions subject to thermal fluctuations was presented based on a mechanical description utilizing both Eulerian and Lagrangian reference frames. General conditions were established for operators coupling these descriptions. Reduced descriptions for the stochastic dynamics of the fluid-structure system were developed for several physical regimes. Analysis was presented for each regime establishing for the SELM stochastic dynamics that the Gibbs-Boltzmann distribution is invariant. The SELM formalism provides a general framework for the development of computational methods for applications requiring a consistent treatment of structure mechanics, hydrodynamic coupling, and thermal fluctuations.

8. Acknowledgements. The author P.J.A. acknowledges support from research grant NSF CAREER DMS - 0956210.

\section{REFERENCES}

[1] D. J. Acheson, Elementary Fluid Dynamics, Oxford Applied Mathematics and Computing Science Series, 1990.

[2] B. Alberts, A. Johnson, J. Lewis, M. Raff, K. Roberts, and P. Walker, Molecular Biology of the Cell, Garland Publishing, 2002.

[3] M. Arienti, P. Hung, E. Morano, and J. E. Shepherd, A level set approach to eulerianlagrangian coupling, Journal of Computational Physics, 185 (2003), pp. 213-251-.

[4] P. Atzberger, Spatially adaptive stochastic multigrid methods for fluid-structure systems with thermal fluctuations, (preprint), . (2010), p. .

[5] P.J. AtZBerger, Spatially adaptive stochastic numerical methods for intrinsic fluctuations in reaction-diffusion systems, Journal of Computational Physics, 229 (2010), pp. 3474-3501.

[6] P. J. Atzberger, P. R. Kramer, and C. S. Peskin, A stochastic immersed boundary method for fluid-structure dynamics at microscopic length scales, Journal of Computational Physics, 224 (2007), pp. 1255-1292-.

[7] A. J. BANChio AND J. F. BRAdy, Accelerated stokesian dynamics: Brownian motion, Journal of Chemical Physics, 118 (2003), pp. 10323-10332-.

[8] D. Bedeaux and P. Mazur, Brownian motion and fluctuating hydrodynamics, Physica, 76 (1974), pp. 247-258.

[9] Garcia A. L. Williams S. A. Bell, J. B, Computational fluctuating fluid dynamics, ESAiM: Mathematical Modelling and Numerical Analysis., . ((to appear)), p. .

[10] N. Bodard And M. O. Deville, Fluid-structure interaction by the spectral element method, Journal of Scientific Computing, 27 (2006), pp. 123-136.

[11] J. F. BRAdy AND G. Bossis, Stokesian dynamics, Annual review of fluid mechanics. Vol.20-Annual review of fluid mechanics. Vol.20, (1988), pp. 111-57.

[12] L. Braescu and T. F. George, Arbitrary lagrangian-eulerian method for coupled navierstokes and convection-diffusion equations with moving boundaries, Applied Mathematics for Science and Engineering, (2007), pp. 31-36.

[13] Fisher J. E. Roma. A. M. Ceniceros, H. D., Efficient solutions to robust, semi-implicit discretizations of the immersed boundary method, J. Comput. Phys.,, 228 (2009), pp. 71377158.

[14] Yong Chen, Nitin Sharma, and Neelesh Patankar, Fluctuating Immersed Material (FIMAT) Dynamics for the Direct Simulation of the Brownian Motion of Particles, vol. 81, Springer Netherlands, 2006.

[15] Valentine M.T. Weeks E.R. Gisler T.. Kaplan P.D.. Yodh A.G.. Crocker, J.C. and Weitz. D.A., Two-point microrheology of inhomogeneous soft materials, Physical Review Letters, 85 (2000), p. .

[16] G. Da Prato And J. ZabczyK, Stochastic Equations in Infinite Dimensions, Cambridge University Press, 1992. 
[17] Gaudenz Danuser and Clare M Waterman-Storer, Quantitative fluorescent speckle microscopy of cytoskeleton dynamics., Annu Rev Biophys Biomol Struct, 35 (2006), pp. 361387.

[18] G. De Fabritis, M. Serrano, R. Delgado-Buscalioni, and P. V. Coveney, Fluctuating hydrodynamic modeling of fluids at the nanoscale, Phys. Rev. E, 75 (2007), pp. 026307-.

[19] M. Doi and S. F. Edwards, The Theory of Polymer Dynamics, Oxford University Press, 1986.

[20] A. Donev, J. B. Bell, A. L. Garcia, And B. J. Alder, A hybrid particle-continuum method for hydrodynamics of complex fluids, SIAM J. Multiscale Modeling and Simulation, 8 (2010), pp. 871-911.

[21] A. Donev, E. Vanden-Eijnden, A. L. Garcia, and J. B. Bell, On the accuracy of finitevolume schemes for fluctuating hydrodynamics, ArXiv e-prints, 0906,2425 (2009), p. .

[22] E.H. Dowell ANd K.C. Hall, Modeling of fluid-structure interaction, Ann. Rev. Fluid Mech., 33 (2001), pp. 445-490.

[23] D. L. ERmak and J. A. McCammon, Brownian dynamics with hydrodynamic interactions, J. Chem. Phys., 69 (1978), pp. 1352-1360.

[24] Y.C. Fung, An Introduction to the Theory of Aeroelasticity, John Wiley, 1955.

[25] C. W. Gardiner, Handbook of stochastic methods, Series in Synergetics, Springer, 1985.

[26] I. M. Gelfand And S. V. Fomin, Calculus of Variations, Dover, 2000.

[27] E Givelberg and J Bunn, A comprehensive three-dimensional model of the cochlea, JOURNAL OF COMPUTATIONAL PHYSICS, 191 (2003), pp. 377-391.

[28] R. Gotter, K. Kroy, E. Frey, M. Barmann, and E. Sackmann, Dynamic light scattering from semidilute actin solutions: A study of hydrodynamic screening, filament bending stiffness, and the effect of tropomyosin/troponin-binding, Macromolecules, 29 (1996), pp. 30-36.

[29] D. Gottlieb and S. A. Orszag, Numerical Analysis of Spectral Methods Theory and Applications, SIAM Publishing, 1993.

[30] B.E. Griffith, X. Luo, D.M. McQueen, And C.S. Peskin., Simulating the fluid dynamics of natural and prosthetic heart valves using the immersed boundary method., Int J Appl Mech., 1 (2009), pp. 137-177.

[31] E. H. Hauge and Martin-Loff, Fluctuating hydrodynamics and brownian motion, Journal of Statistical Physics, 7 (1973), pp. 259-281.

[32] B.P. HILls, A generalized langevin equation for the angular velocity of a spherical brownian particle from fluctuating hydrodynamics, Physica A: Statistical Mechanics and its Applications, 80 (1975), pp. 360-368.

[33] Yongsam Kim and Charles S. Peskin, Penalty immersed boundary method for an elastic boundary with mass, Phys. Fluids, 19 (2007), pp. 053103-18.

[34] Kloeden.P.E. AND E. Platen, Numerical solution of stochastic differential equations, Springer-Verlag, 1992.

[35] P. R. KRAmer and A. J. Majda, Stochastic mode reduction for immersed boundary method, SIAM J. Appl. Math., 64 (2003), pp. 369-400.

[36] L.D. Landau AND E. M. Lifshitz, Course of theoretical physics, vol. 9: statistical physics, Pergamon Press, Oxford, 1980.

[37] R. G Larson, The Structure and Rheology of Complex Fluids, Oxford University Press, 1999.

[38] ERIC LaUga And Thomas R Powers, The hydrodynamics of swimming microorganisms, 2009.

[39] V.I. Lebedev and D.N. Laikov, A quadrature formula for the sphere of the 131st algebraic order of accuracy., Doklady Mathematics, 59 (1999), pp. 477-481.

[40] E.H. Lieb and M. Loss, Analysis, American Mathematical Society, 2001.

[41] Sookkyung Lim, Anca Ferent, X. Sheldon Wang, and Charles S. Peskin, Dynamics of a closed rod with twist and bend in fluid, SIAM J. Sci. Comput., 31 (2008), pp. 273-302.

[42] P. Mazur and D. Bedeaux, A generalization of faxn's theorem to nonsteady motion of a sphere through an incompressible fluid in arbitrary flow, Physica, 76 (1974), pp. 235-246.

[43] F. Mezei, C. Pappas, and T. Gutberlet, Neutron spin echo spectroscopy: basics, trends, and applications, Spinger-Verlag, 2003.

[44] L. A. Miller And C. S. Peskin, A computational fluid dynamics of 'clap and fing' in the smallest insects, Journal of Experimental Biology, 208 (2005), pp. 195-212-.

[45] Rajat Mittal and Gianluca Iaccarino, Immersed boundary methods, Annual Review of Fluid Mechanics, 37 (2005), pp. 239-261.

[46] Jeffrey R. Moffitt, Yann R. Chemla, Steven B. Smith, and Carlos Bustamante, Recent advances in optical tweezers, Annual Review of Biochemistry, 77 (2008), pp. 205-228.

[47] Eijkel. J. C. T. NAPoli, M. And S. Pennathur, Nanofluidic technology for biomolecule applications: a critical review, Lab on a Chip, 10 (2010), pp. 957-985.

[48] B. OKsendal, Stochastic Differential Equations: An Introduction, Springer, 2000. 
[49] C. S. PESKIn, Numerical analysis of blood flow in the heart, Journal of Computational Physics, 25 (1977), pp. 220-252.

[50] — The immersed boundary method, Acta Numerica, 11 (2002), pp. 479-517.

[51] E. M. Purcell, Life at low reynolds number, American Journal of Physics, 45 (1977), pp. 3-10.

[52] R. C. Armstrong R. Byron Bird and O. Hassager, Dynamic Polymeric Liquids, Vol. I and Vol. II, John Wiley \& Sons, 1987.

[53] Michael Reichert and Holger Stark, Hydrodynamic coupling of two rotating spheres trapped in harmonic potentials, Phys. Rev. E, 69 (2004), pp. 031407-.

[54] L. E. ReIchl, A Modern Course in Statistical Physics, John Wiley and Sons, 1998.

[55] Jens Rotne and Stephen Prager, Variational treatment of hydrodynamic interaction in polymers, J. Chem. Phys., 50 (1969), pp. 4831-4837.

[56] David Saintillan, Eric Darve, and Eric S. G. Shaqfeh, A smooth particle-mesh ewald algorithm for stokes suspension simulations: The sedimentation of fibers, Phys. Fluids, 17 (2005), pp. 033301-21.

[57] Todd M. Squires And Stephen R. Quake, Microfluidics: Fluid physics at the nanoliter scale, Rev. Mod. Phys., 77 (2005), pp. 977-.

[58] G. Strang, Linear Algebra and its Applications, Harcourt Brace Jovanovich College Publishers, 1988.

[59] G. Strang and G. Fix, An Analysis of the Finite Element Method, Wellesley-Cambridge Press, 2008

[60] J. C. Strikwerda, Finite Difference Schemes and Partial Differential Equations, SIAM Publishing, 2004.

[61] L. N. Trefethen and D. Bau, Numerical Linear Algebra, Society for Industrial and Applied Mathematics, 1997.

[62] M. E. Tuckerman, C. J. Mundy, and G. J. Martyna, On the classical statistical mechanics of non-hamiltonian systems, EPL (Europhysics Letters), 45 (1999), pp. 149-155.

[63] X. S. Wang, L. T. Zhang, And W. K. Liu, On computational issues of immersed finite element methods, Journal of Computational Physics, 228 (2009), pp. 2535-2551-.

[64] Nobuhiko Watari, Masao Doi, and Ronald G. Larson, Fluidic trapping of deformable polymers in microflows, Phys. Rev. E, 78 (2008), pp. 011801-.

[65] Max C. Watson ANd Frank L.H. Brown, Interpreting membrane scattering experiments at the mesoscale: The contribution of dissipation within the bilayer, Biophysical Journal, 98 (2010), pp. L9-L11.

[66] Hiromi Yamakawa, Transport properties of polymer chains in dilute solution: Hydrodynamic interaction, J. Chem. Phys., 53 (1970), pp. 436-443.

[67] T. I. Zohdi And P. Wriggers, Introduction to Computational Micromechanics, Springer, 2005. 